\title{
Better than average: Alternatives to statistical summary representations for rapid judgments of average size
}

\author{
Kristoffer MycZeK ANd DANiEl J. Simons \\ University of Illinois at Urbana-Champaign, Champaign, Illinois
}

\begin{abstract}
People can perceive the individual features of an object by focusing attention on it and binding the features together at a location. Some perceptual processing can occur without focusing attention on each object, though; people may even be able to extract summary information about the sizes of all the objects in a display, essentially computing the mean size at a glance. Evidence that people can judge the mean size of an array efficiently and accurately has been used to support the strong claim that people use a global, parallel process to extract a statistical summary of the average size of the objects in the display. Such claims are based both on the accuracy of performance and on the supposition that performance exceeds what would be possible with serial, focused attention. However, these studies typically have not examined the limits of performance with focused-attention strategies. Through experiments and simulations, we show that existing evidence for mean size perception can be explained through various focused-attention strategies, without appealing to a new mechanism of average size perception. Although our evidence does not eliminate the possibility that people do perceive the average size of all the objects in a display, it suggests that simpler mechanisms can accommodate the existing data.
\end{abstract}

Human observers rapidly extract the gist or meaning of visual scenes, seemingly with little effort. Yet the capacity of visual working memory and of attention appears to be limited to a few objects. Extensive evidence for change blindness and other related failures of awareness suggests that observers take in far less of their visual world than we might expect on the basis of their ability to perceive and remember visual scenes. Together, these findings suggest that gist perception might not rely on the same mechanisms as those responsible for object recognition, feature binding, and visual working memory. For gist perception, one alternative might be the use of global characteristics of the scene, such as the spatial frequency amplitude spectrum or the distribution of colors in an image (Oliva \& Schyns, 2000; Oliva \& Torralba, 2001). By relying on global scene properties that can be processed in parallel, rather than on individual objects, the visual system could avoid the limitations imposed by the need for attention to bind and retain individual visual objects in working memory. Such statistical representations of image features might well underlie our ability to perceive and discriminate the gist of scenes with brief presentations and minimal attentional resources.

In some cases, observers can perceive a summary representation of many elements in a display in parallel to determine an overall property of that display. For example, observers can determine the overall movement direction of a set of dots moving in different directions and at different speeds (Watamaniuk \& Duchon, 1992); people seem to per- ceive the entire display on the basis of a statistical summary of the individual elements in the display, without focusing much attention on the individual elements. They appear to do so in parallel across the display. Similarly, people can perceive the average orientation of a set of gratings even when the individual gratings are hard to individuate due to crowding (Parkes, Lund, Angelucci, Solomon, \& Morgan, 2001). In both cases, perception of the overall pattern in the display is, in some respects, similar to texture perception (Cavanagh, 2001). In essence, the sensory signals for motion or orientation are pooled across the visual display, leading to a single percept of the predominant motion or orientation in the display. Note that in these cases, the percept could result from pooled responses of individual receptors that operated in parallel, rather than from focused attention to the motion or orientation of each element.

More recent evidence from a variety of tasks suggests that people can also perceive the average size of an array of circles even when they typically cannot recall the sizes of individual elements in the array (Ariely, 2001; Chong \& Treisman, 2003, 2005a, 2005b). For example, observers can achieve $75 \%$ accuracy in judging the average size of an array of objects to a fairly high degree of precision $(4 \%-12 \%)$, even when the array is visible for only $500 \mathrm{msec}$ (Ariely, 2001). And performance is relatively unaffected by increases in the number of elements in the array (Ariely, 2001), the variability of the elements in the array (Chong \& Treisman, 2003), or the presentation duration

D. J. Simons, dsimons@uiuc.edu 
of the display (Chong \& Treisman, 2003). These findings have been taken as evidence that observers rapidly compute a statistical summary representation (i.e., the mean) of the size of the individual elements in the array and that they do so without relying on focused attention (Ariely, 2001; Chong \& Treisman, 2003, 2005a, 2005b); they process the display in parallel, extracting the average size globally without focusing attention on each object individually.

The claim that observers perceive the average size of the items in a display in parallel is interestingly different from claims that they perceive the predominant motion or orientation of a set of items. For motion or orientation, pooling across a set of receptors that specialize for different motion directions or orientations could lead, in a straightforward way, to a coherent percept of the average motion or orientation. And such a mechanism could operate largely preattentively and in parallel. Unlike for motion or orientation, though, there are no individual receptors in the early visual system that are specific to different absolute sizes of objects, so it is less clear what could be pooled to produce a percept of the mean size. Moreover, processing of absolute object size is traditionally thought to require focused attention (at least for subtle discriminations of object sizes), and evidence that observers rapidly perceive the average size of an array of circles suggests the need for a new averaging mechanism that relies minimally on focused attention.

The possibility that observers may be computing the mean size of an array of objects in parallel has striking implications for scene perception. If observers can use such statistical averaging processes for features of individual objects, limits on the capacity of focused attention might not impose the strict limits on perceptual awareness that are often assumed in studies of change blindness and visual attention. Such processes might even provide a mechanism for implicit change detection by, for example, providing a difference signal in the absence of focused attention. That is, observers might be able to detect changes to the statistical summary representation without detecting a change to any of the individual elements in the array. It might also provide a mechanism for proposed phenomena such as "mindsight," the idea that people sometimes sense a change before they visually experience and localize it (Rensink, 2004). And it might help bridge the gap between models of slow visual search processes and the faster mechanisms that presumably underlie the rapid perception of scene meaning (e.g., Potter, 1976). Thus, strong evidence for the perception of a statistical summary representation for an object feature that appears integral to that object (e.g., its size) has striking implications for scene perception and memory in general. These implications help explain why evidence for average size perception has garnered so much interest in the visual cognition literature. ${ }^{1}$

The claim that a new averaging mechanism is needed to explain how people perceive the average size of an array of items is based on three types of evidence: (1) Performance is so efficient that it would exceed the limits of focused attention; (2) performance is so accurate that it would exceed the limits of focused attention; and (3) performance on an average size perception task differs from performance on a recognition memory task for the individual items.
Type 1 is based on evidence that average size perception is relatively unaffected by the presentation duration of the array or the number of objects in the array. Given the limited speed of focused attention (Wolfe, Alvarez, \& Horowitz, 2000), subjects should be unable to focus attention on all the objects in an array when given a limited presentation duration, and focused attention typically is affected by the number of items in an array (Treisman \& Gelade, 1980). The conclusion of global or parallel averaging is based on the idea that subjects lack the time to inspect each item with focused attention but they still perform the task efficiently. Note that this conclusion implicitly assumes that the only focused-attention alternative to a new averaging mechanism is that observers inspect all items in a display with focused attention.

Type 2 is based on evidence that subjects can perceive the average size with approximately $75 \%$ accuracy with a precision of $4 \%-12 \%$ (Ariely, 2001). Such accurate performance suggests that a dedicated mechanism may contribute to the perception of statistical summary representations.

Type 3 is based on evidence that performance when observers try to perceive the average size of a set of objects often exceeds their performance when trying to recognize individual objects from the same set. Perhaps more important, perceiving the mean of a display appears to be enhanced by forcing observers to distribute attention rather than to focus it, presumably allowing the observers to take in more of the display at once (Chong \& Treisman, 2005a). In contrast, recognizing individual elements from an array is relatively enhanced when subjects are forced to focus attention rather than distribute it.

Whenever a new mechanism is proposed that challenges established views of perception and attention, parsimony demands that alternative mechanisms that are consistent with existing theory be systematically eliminated. Evidence of Type 1 is based on the efficiency of performance, but the claim that focused attention is insufficient does not follow from accurate performance. Performance might well be too efficient to permit an exhaustive, serial inspection of all the objects in the display. If subjects can perform a task without calculating an average of every item in the display, however, they might well be able to make their judgments without exceeding the limits imposed by the capacity of focused attention. If so, the inference of a new averaging mechanism is weakened. Critically, if subjects can perform a judgment task by focusing attention on a small subset of items in the set, the number of items in the set and the duration of presentation will have minimal effect on performance.

Evidence of Type 2 is based solely on the face validity of the claim that performance seems too accurate to accommodate the limited capacity of focused-attention mechanisms. Yet none of the published studies directly examined how well subjects might do when adopting various focused-attention strategies. Overall accuracy levels also vary across studies and methods, raising questions about just how good performance would have to be to justify claims of a parallel averaging process.

Evidence of Type 3 is based on dissociations between perceiving the average size of a set of objects and remembering the sizes of individual objects within the set. Such 
dissociations support the claim that performance on these tasks differs, but the dissociation might result from the different demands of these two tasks on focused attention, rather than on a distinction between a focused-attention and a global, parallel mechanism.

In this article, we reexamine all three types of evidence used to claim support for a global, parallel average size perception mechanism, and we argue that all of the extant results are consistent with focused-attention mechanisms. That is, we argue that existing evidence does not necessitate a new averaging mechanism. As an existence proof that focused-attention strategies can produce performance comparable to that obtained in published studies, we modeled the performance of an ideal observer using various focused-attention strategies. We found that such strategies are sufficient to account for the original results, implying that existing evidence for statistical averaging of object size does not necessitate a global, parallel average size perception mechanism. Although performance is consistent with statistical summary representations, it is also consistent with a capacity-limited, serial, focusedattention mechanism. ${ }^{2}$

\section{Methods Used to Study Average Size Perception}

In most studies of average size perception, one of two different approaches has been used. In the first published experiments (Ariely, 2001), subjects reviewed an array of disks followed by a single test probe, and they reported whether the test probe was bigger or smaller than the average size of the elements in the array. In this task, the subjects showed approximately $75 \%$ correct judgments when the size of the probe differed from the mean for the test array by $4 \%-12 \%$. In Experiment 1, we replicated this original demonstration of statistical averaging of size (Ariely, 2001), using similar methods. We then simulated the expected performance, using a simple focused-attention strategy. In the second experiment, we used the same task but decreased the regularity of the distribution of element sizes within each array. Given some of the subtle differences between our experiments and the original experiment (Ariely, 2001), we also simulated the displays and task used in the original experiment and examined whether focused-attention strategies can explain the original data as well. We also simulated performance in another recent variant of this array/probe task (Chong \& Treisman, 2005a).

More recent studies of average size perception (Chong \& Treisman, 2003, 2005b) have used a forced choice method in which observers view two arrays of disks and judge which array has the larger average size. Again, observers perform well in this task, correctly selecting the array with the larger average much of the time $(75 \%)$, with relatively small differences between the arrays $(6 \%-12 \%)$. Performance with this task is largely unaffected by variations in the display time and distribution of sizes in the arrays. We also simulated this task to explore whether focusedattention strategies can produce performance comparable to that achieved in the experiments.

A final task used to study average size perception involves the method of adjustment; subjects view an array of disks followed by a test probe, and they adjust the size of the probe to match the mean of the array. To our knowledge, the only published study using this method was a pilot experiment that was performed to determine whether judgments of mean size were based on the diameter or the area of the disks (Chong \& Treisman, 2003). The method is promising because it requires observers to estimate the mean size directly, rather than making a dichotomous judgment. In essence, it is more like a recall task than a recognition task, and observers cannot use information from a foil or from the test probe to help make their judgments. Given that no published studies have used this method to infer an averaging mechanism, though, we do not address it in this article. ${ }^{3}$

Although our goal is to demonstrate that focusedattention explanations are sufficient to explain existing evidence for average size judgments, our results do not exclude the possibility that observers do perceive the average size of the items in the display. Our purpose is to demonstrate that existing evidence does not necessitate a global, parallel averaging mechanism, because performance does not exceed the capacity of focused attention. To the extent that our proposed alternatives are plausible and are consistent with extant evidence for the role of focused attention in object perception, the justification for positing a global, parallel averaging mechanism is weakened. Arguments for parsimony are always open to debate: Which view is simpler or more plausible? It seems prudent, though, to eliminate known mechanisms in order to support the existence of new ones.

\section{EXPERIMENT 1}

This experiment was designed as a conceptual replication of the forced choice probe method (Ariely, 2001) in which observers view an array of circles and then decide whether a single test probe is bigger or smaller than the mean of the objects in the array. The methods are based on those used by Ariely, with some minor differences. Given that the pattern of results matches those from the initial study, these differences are not critical to our results or simulations. To verify that the results of our experiments are comparable to those of Ariely, after Experiments 1 and 2, we also simulated the original displays and tasks used in that study.

\section{Method}

Subjects. Nineteen University of Illinois undergraduates participated in exchange for course credit. All had self-reported normal or corrected-to-normal vision.

Materials and Procedure. The task was controlled using VisionEgg software on Macintosh eMac computers with a 17-in. display and resolution set to $1,024 \times 768$. The subjects sat approximately $57 \mathrm{~cm}$ from the display, so that $1 \mathrm{~cm}$ on the display equaled $1^{\circ}$ of visual angle. Head position was loosely constrained by a set of headphones that were tethered to the monitor. The stimuli were white filled circles presented against a gray background.

On each trial, the observers first viewed an array of eight circles for $500 \mathrm{msec}$ and then viewed a test circle until their response (see Figure 1 for the trial sequence). The task was to judge whether the average size of the eight circles was larger or smaller than that of the test circle. The circles in the array were positioned in a rectangular grid with three rows. The top and bottom rows had three slots for objects, and the middle row had two (see Figure 2). One object was entirely contained within each slot, and the position within the slot was otherwise 


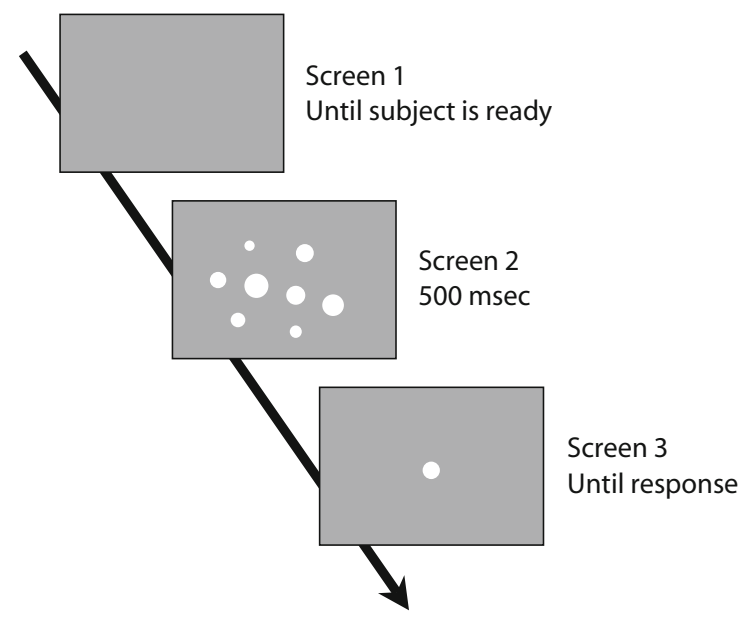

Figure 1. Sequence of trials in both experiments (not drawn to scale).

randomly determined, thereby making the array layout more variable across trials. The entire array subtended $18^{\circ} \times 10^{\circ}$. Across trials, the circles varied in size, with diameters ranging from $1.13^{\circ}$ to $3.17^{\circ}$. Within a trial, the range of possible circle sizes was constrained to a subset of that larger range, so that trials had different averages. Fortyone different size ranges were used. Following Ariely's (2001) design, for each array, the sizes of the items in the array were determined in a stepwise fashion, with a $10 \%$ size difference between each element (Ariely's study used 5\% or $40 \%$ increments; see Simulation 1 below).
Unlike Ariely, we used eight different object sizes in each array and only used arrays with eight items. For example, the array with the smallest circles had a range of $1.13^{\circ}-2.19^{\circ}$ and included circles with the following diameters: $1.13^{\circ}, 1.24^{\circ}, 1.37^{\circ}, 1.50^{\circ}, 1.65^{\circ}, 1.82^{\circ}, 2.00^{\circ}$, and $2.19^{\circ}$. Thus, the size of the range within a display varied, but the distribution of the relative sizes within the array was constant, with $10 \%$ differences between each element size. Successively larger size ranges started with a smallest item that was $0.0125^{\circ}$ larger than the smallest item in the previous range (e.g., Range 1 started with $1.13^{\circ}$, Range 2 with $1.1425^{\circ}$, Range 3 with $1.155^{\circ}$, etc., up to $1.63^{\circ}$ for the largest range). The size range on each trial was randomly selected from the 41 possible ranges.

After the array disappeared, a test circle appeared, and the subjects were instructed to compare the size of the test circle with the average size of the array. If they judged the test circle to be larger than the mean of the array, they pressed the up arrow key. If smaller, they pressed the down arrow key. The test circle was either bigger or smaller than the mean size of the prior array by $1 \%-20 \%$, in $1 \%$ increments. Consequently, there were 41 possible test circles for each array mean, with size discrepancies ranging from $20 \%$ smaller to $20 \%$ larger. For 1 out of every 41 trials, the test circle had the same size as the mean of the array (a $0 \%$ change), so neither answer was correct. These trials served to assess a bias in the tendency to respond larger or smaller.

Each subject completed 15 trials with each of the 41 relative sizes of the test circle $(-20$ to +20$)$, with the size range on each trial being determined randomly. Thus, each subject completed a total of 615 trials $(41 \times 15)$, which took approximately $30-50 \mathrm{~min}$. They were allowed to take breaks between trials as necessary.

\section{Results and Discussion}

Consistent with the original results (Ariely, 2001), the subjects accurately judged the relationship between the

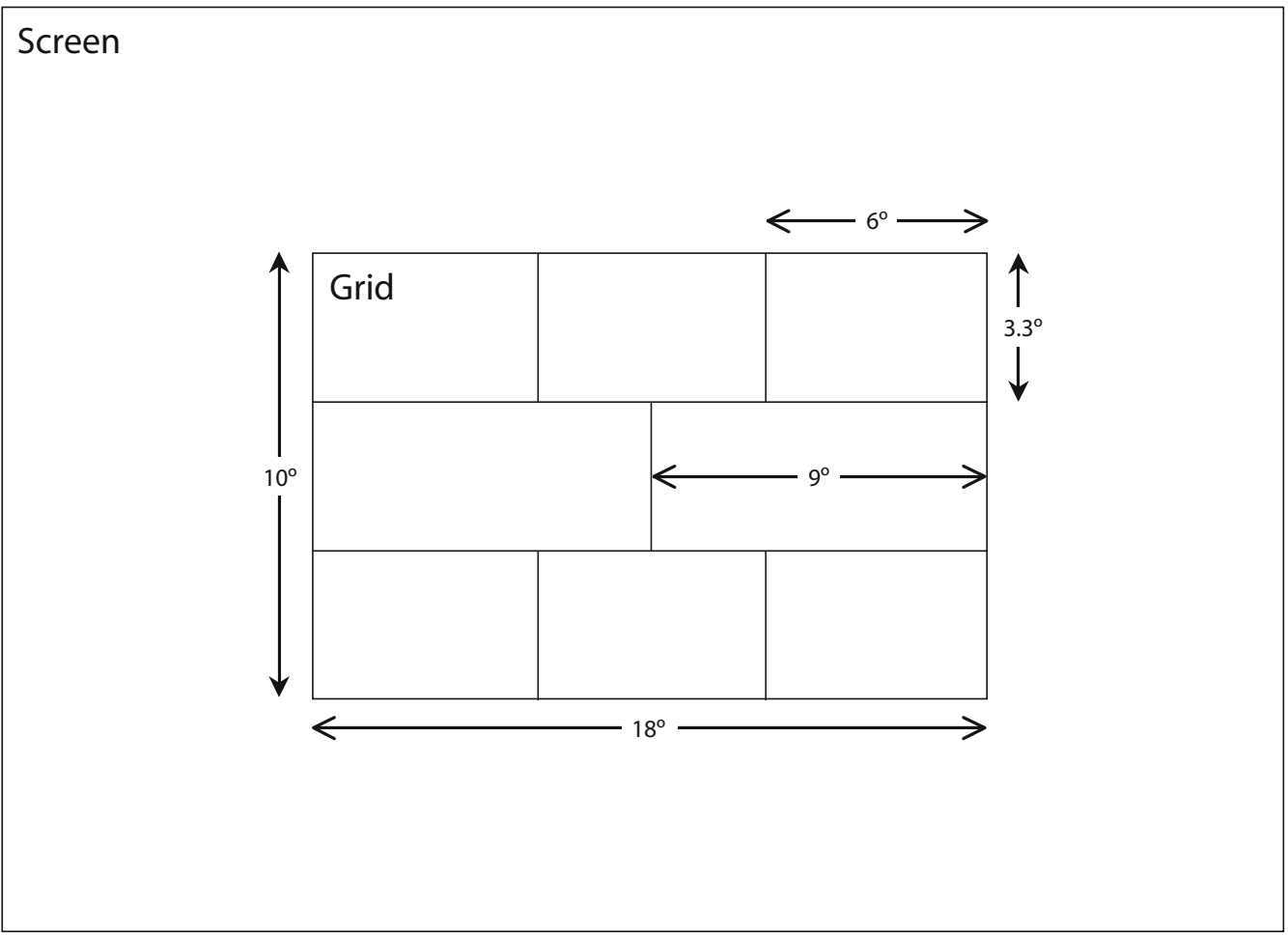

Figure 2. Possible array positions and variability (not drawn to scale). 
mean size in the array and the size of the test probe when the size difference between the probe and the array was large enough (see Figure 3). Across all size differences (i.e., the difference in size between the test probe and the mean of the array), the subjects were correct $69.3 \%$ of the time. Performance was at chance with a $1 \%$ size difference $(M=50.4 \%)$ and approached $85 \%$ correct with a $20 \%$ size difference $(M=83.7 \%)$. With a size difference of $13 \%$ or greater, accuracy consistently exceeded $75 \%$ correct. These results appear comparable to Ariely's evidence that discrimination thresholds fell between $4 \%$ and $12 \%$, depending on the stimuli.

To determine whether this pattern of results requires a statistical summary representation, we simulated the anticipated performance for various focused-attention strategies. If focused attention is limited to four or fewer items (e.g., Rensink, 2000), subjects could, in principle, sample a small subset of items with focused attention and make their judgment on the basis of the mean size of that small subset. Such a strategy would not involve the perception of the mean size of the entire array. If performance is entirely consistent with such a strategy, there would be no need to posit a new mechanism for perceiving the average size of an array. We simulated the expected accuracy levels on the basis of the assumption that the subjects simply selected one, two, or three items in the array at random and compared the mean of those items with the test probe to make their judgment. In principle, given that object locations within the array were randomly determined, selecting one item at random would be equivalent to selecting the item closest to fixation, which would be a more likely strategy for an actual subject. Consistent with this idea, during debriefing, a number of the subjects reported adopting a strategy of focusing on the middle circles.
For each strategy simulation (sample one, sample two, or sample three), we generated 13,448 trials for each size difference ( -20 to +20 , excluding 0 ), for a total of 537,920 trials for each simulation. For each trial of the sample one simulation, we calculated the probability of a correct response based on randomly selecting one item from the display and comparing that item with the test probe. For the sample two and sample three simulations, we randomly selected two or three items from the array, computed the average diameter of those items, and compared that average with the test probe diameter. ${ }^{4}$ For all the simulations, we assumed that the comparison process was infallible, so that even the smallest difference between the test stimulus and the selected item (or the mean of the selected items in the select two and select three conditions) generated the appropriate response. Of course, people do make errors in their judgments, so this analysis represents an ideal observer and suggests the maximum performance possible with this strategy. The results of the simulation are presented in Figure 3.

Note that the effectiveness of selecting a subset of the items in an array varies with the size difference between the test stimulus and the mean of the array. With just a 7\% size difference between the test stimulus and the mean of the array, five of the eight items would individually produce a correct response. Thus, sampling one item at random would produce accurate responses on $62.5 \%$ of the trials. With a $16 \%$ size difference, six of the eight items would individually give a correct response ( $75 \%$ correct).

Averaging across the size differences, a strategy of sampling one item from the array at random and comparing that item with the test probe produced a simulated accuracy of $64.1 \%$. Sampling two items at random from the array produced an average accuracy of $73.7 \%$ correct, bet-

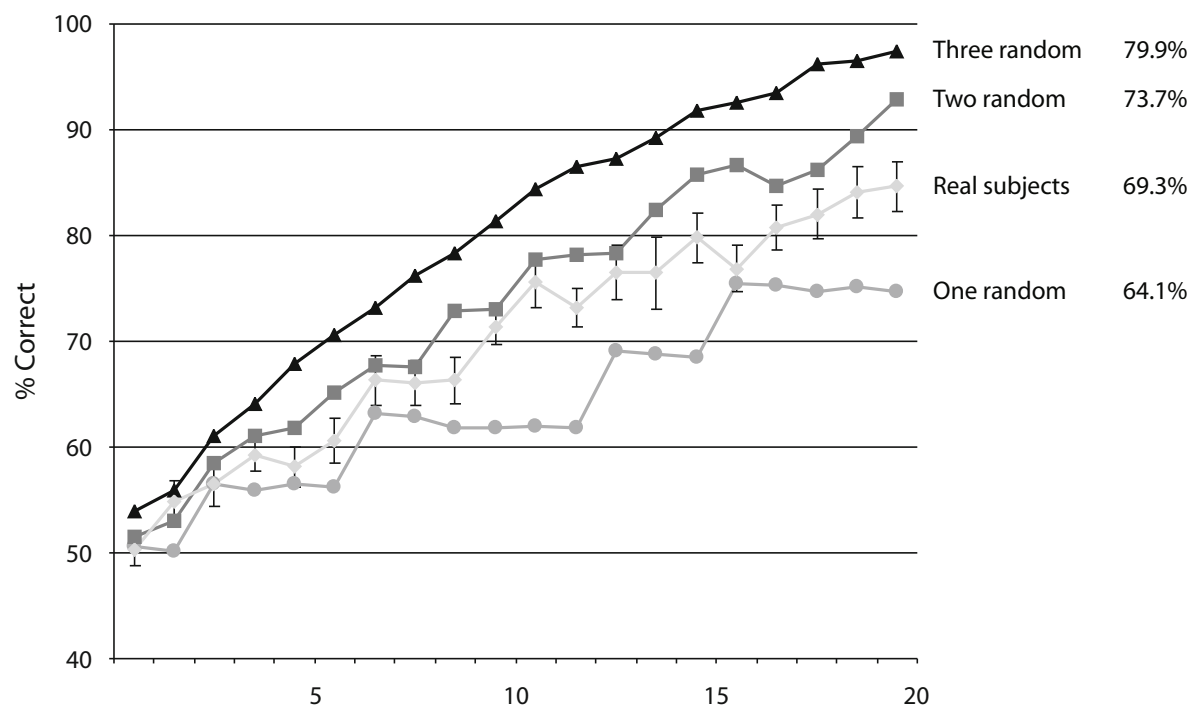

\% Size Difference Between Group Average and Test Circle

Figure 3. Data from Experiment 1, together with simulations of the select one, select two, and select three strategies. 
ter than the actual level of performance! Sampling three items produced an average accuracy of $79.9 \%$ correct. Thus, average performance across the range of size differences used in our experiment is consistent with randomly sampling between one and two items at $\operatorname{random}(M=1.54$ items, equivalent to selecting one item on approximately $45 \%$ of the trials and two random items on approximately $55 \%$ of the trials) and making the judgment entirely on the basis of that subset of items. Such a strategy is computationally simpler than averaging the sizes of all the display elements and is consistent with established limits on the capacity of focused attention. Subjects could sample any one or two items close to fixation and compare those with the test stimulus.

We also examined how well these strategies fit the performance of each subject. For each subject, we calculated the sum of the mean squared errors between their accuracy performance and the accuracy prediction for each strategy at each level of size difference $(1 \%-20 \%)$. Most of the subjects were matched best by the strategy of selecting one $(42.1 \%$ of the subjects) or two $(47.4 \%)$ items at random. Far fewer subjects were characterized well by chance performance $(5.26 \%$; i.e., $50 \%$ accuracy at each size difference) or by selecting three items at random (5.26\%). Thus, performance in this task does not require taking an average of all items in the array. Simply selecting one or two items at random produces performance comparable to that found in studies claiming support for a statistical summary representation. Of course, subjects might still perceive the average, but this simulation provides a plausible alternative that does not require a new mechanism.

\section{EXPERIMENT 2}

The distributions of object sizes in the arrays in Experiment 1 were designed to replicate those used by Ariely (2001). In Experiment 1, item sizes in each array were determined in a stepwise fashion, so that each object was $10 \%$ bigger than the next smallest one. Given this regularity, the strategy of sampling one or two items at random produces reasonably accurate performance. In Experiment 2, we randomly determined the sizes of objects within each array, with the constraint that the range and variance of sizes for each array were approximately the same as those of the uniform distribution used in Experiment 1 (within $2 \%$ ). Decreasing the regularity of the arrays in this fashion should slightly decrease the accuracy obtained by selecting one item at random from the array, because the number of items that would produce a "correct" response on their own would be reduced (i.e., items sometimes would cluster on the "wrong" side for the judgment, with an outlier in the "correct" direction compensating for the change to the mean and variance). For example, with a $7 \%$ size difference between the test probe and the mean of the array, five out of eight items $(62.5 \%)$ individually would produce the correct answer with a uniform distribution, but only four and one half items, on average (56.8\%), would do so in the random distribution.

Thus, with a random distribution, the size of any individual object is less predictive of the mean of the entire array (although selecting one item at random still would be an effective strategy). Consequently, if subjects use a strategy of sampling one item to make their judgment, their accuracy should be slightly worse in Experiment 2 than it was in Experiment 1. Note that averaging based on the entire set of objects in the array should be unaffected by the nature of the distribution.

\section{Method}

Subjects. Nineteen University of Illinois undergraduates with self-reported normal or corrected-to-normal vision participated in Experiment 2 in exchange for course credit.

Materials and Procedure. Except as noted, all materials and procedures were identical to those in Experiment 1. The sizes of the eight objects in each array were determined randomly, with the constraint that the range and variance were nearly identical (within $2 \%$ ) to those in Experiment 1 for that range of sizes. So, for example, in the smallest size range $\left(1.13^{\circ}-2.19^{\circ}\right)$, the eight circles still had a variance of .12 , but their individual sizes were otherwise randomly determined.

\section{Results}

The random distributions in Experiment 2 led to somewhat less accurate performance overall $(M=65.8 \%)$ than in Experiment $1(M=69.3 \%)$, although the difference was not significant with a two-tailed test $[t(36)=1.608, p=.1164$; see Figure 4]. In Experiment 2, the subjects achieved performance of $75 \%$ correct only for the $19 \%$ size difference.

We simulated the effectiveness of sampling one, two, or three items at random in the same manner as in Experiment 1 . As was expected, the predictive value of selecting one item at random was reduced from $64.1 \%$ in Experiment 1 to $61.8 \%$ in Experiment 2. The predictive value of sampling two or three items at random was comparable to the prediction from Experiment 1 (less than a 1\% change in predicted accuracy).

The strategy of sampling one item produced the best fit to the data for $63.1 \%$ of the subjects in Experiment 2, more than the $42.1 \%$ in Experiment 1 . This pattern provides additional evidence that the subjects could perform the mean judgment task by sampling one or two items with focused attention and making their comparison on the basis of those items.

\section{DISCUSSION OF EXPERIMENTS 1 AND 2}

Our results replicate the performance curve originally used to support the claim that people can perceive the average size of the objects in an array (Ariely, 2001). As in the earlier study, accuracy increased as the difference between the size of the test circle and the average size of the array increased. However, we also showed, through simulation of our data, that performance in the judgment task can be explained without appealing to the perception of a statistical summary representation. Focusing attention on the closest one or two objects in an array and making judgments on the basis of the sizes of those objects predicts performance comparable to that obtained in our study. This alternative mechanism is consistent with evidence that people show worse memory for individual elements in the arrays than for the average size (Ariely, 2001). If observers sample only a small subset of each array, their 


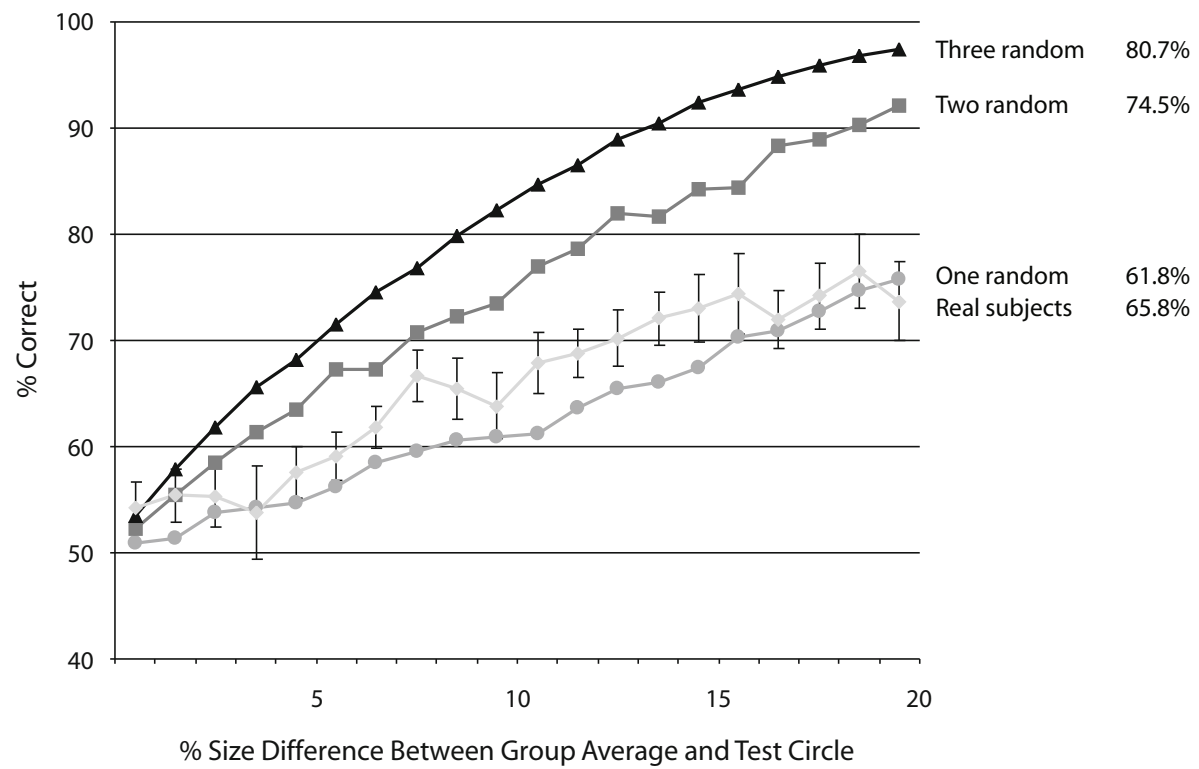

Figure 4. Data from Experiment 2, together with simulations of the select one, select two, and select three strategies.

memory for all the other items in the array will be poor; they simply are not encoded.

Our alternative explanation accounts for performance in this judgment task without appealing to a new sizeaveraging mechanism. No statistical summary representation is needed because observers can perform the task using focused attention. Capacity limits on focused attention are not problematic given that subjects need only attend to one or two items in the array to make their decision. Of course, our results do not disprove the possibility that subjects perform this task by perceiving the mean size. And we have not proven that they do use a strategy of selecting one or two items. Rather, we have provided a plausible alternative to the idea that judging the mean size in this task requires a new perceptual averaging mechanism for size. Further experiments could control for our alternative strategies, in order to provide more convincing evidence for a perceptual size-averaging mechanism or more definitive evidence that people do simply select one or two items in performing this task.

In fact, one new set of findings (de Fockert \& Marchant, 2008) provides converging evidence for our strategybased explanation. ${ }^{5}$ When the subjects were cued in advance to attend to the biggest or smallest item in an array of objects, their judgments of average size were biased in the direction of that item. When cued to the largest item in the array, their judgments were consistent with a larger perceived average; when cued to the smallest item, their judgments were consistent with a smaller perceived average. In other words, when cued to attend to a particular item in the array, judgments of average size were heavily influenced by that item. In their task, rather than the subjects' randomly sampling one item from the array, the cue effectively sampled the array for them, and their judgments were consistent with selecting that item and making the judgment on the basis of it.

Both our results and the de Fockert and Marchant (2008) findings suggest that averaging might not be needed to perform the mean size judgment task with some precision. However, our experimental displays differed from those of Ariely (2001) in several respects: We did not vary the number of objects, we used eight different sizes rather than four, we did not repeat item sizes within a display, and we used only one distribution of sizes $(10 \%$ linear differences between items, rather than $5 \%$ or $40 \%$ differences in $\log$ diameters). To determine whether these factors would alter the effectiveness of our alternative sampling strategy, we simulated performance with display parameters identical to those reported by Ariely. ${ }^{6}$

\section{Simulation 1: Ariely (2001)}

In the original Ariely (2001) study, four different object sizes were used in each array, with a total of $4,8,12$, and 16 objects in each display (i.e., each size was repeated one, two, three, or four times). The items within an array were designed to have a mean diameter of $0.25^{\circ}$ of visual angle and to be evenly distributed on a log scale separated by a factor of either 1.05 or 1.40 . For the smaller scaling factor, the smallest item was $0.23^{\circ}$ and the largest was $0.27^{\circ}$. For the larger scaling factor, the smallest item was $0.15^{\circ}$ and the largest was $0.42^{\circ}$. For our simulations, we used sizes of $0.23^{\circ}, 0.2426^{\circ}, 0.2559^{\circ}$, and $0.27^{\circ}$ for the smaller range (mean size $=0.2496^{\circ}$ ) and $0.15^{\circ}, 0.2114^{\circ}, 0.2980^{\circ}$, and $0.42^{\circ}$ for the larger range (mean size $=0.2698^{\circ}$ ). We simulated performance for a probe that differed from the actual mean size by $1 \%$ through $16 \%$, and we generated 
Table 1

Simulation of the Effectiveness of Using a Sampling Strategy

With Stimuli Approximating Those Using a Smaller Scaling Factor From Ariely (2001)

\begin{tabular}{|c|c|c|c|c|c|c|c|c|c|c|c|c|c|c|c|c|}
\hline \multirow{2}{*}{$\begin{array}{c}\text { Difference } \\
(\%)\end{array}$} & \multicolumn{4}{|c|}{ No. Sampled From 4 Objects } & \multicolumn{4}{|c|}{ No. Sampled From 8 Objects } & \multicolumn{4}{|c|}{ No. Sampled From 12 Objects } & \multicolumn{4}{|c|}{ No. Sampled From 16 Objects } \\
\hline & 1 & 2 & 3 & 4 & 1 & 2 & 3 & 4 & 1 & 2 & 3 & 4 & 1 & 2 & 3 & 4 \\
\hline 16 & 8749 & & & & & & & & & & & & & & & \\
\hline 15 & & & & & & & & & & & & & & & & \\
\hline 14 & & & & & & & & & & & & & & & & \\
\hline 13 & & & & & & & & & & & & & & & & \\
\hline 12 & & & & & & & & & & & & & & & & \\
\hline 11 & & 1.00 & & & & & & & & & & & & & & \\
\hline 10 & & & & & & & & & & & & & & & & \\
\hline & & & & & & & & & & & & & & & & \\
\hline 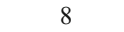 & & & & & & & & & & & & & & & & \\
\hline 7 & & & & & & & & & & & & & & & & \\
\hline 6 & & & & & & & & & & & & & & & & \\
\hline 5 & & .66 & & & .5023 & & & & & & & & & & & .7636 \\
\hline 4 & & & & & & & & & & & & & & & & .7636 \\
\hline 3 & & & & & & & & & & & & & & & & \\
\hline 2 & & & & & & & & & & & & & & & & \\
\hline 1 & .5010 & .6676 & .4996 & 1.0000 & .5023 & .6450 & .5019 & .6289 & .5012 & .6336 & .4972 & .6032 & .5036 & .6335 & .5002 & .5986 \\
\hline
\end{tabular}

20,000 trials for which the probe was smaller than the actual mean and 20,000 trials for which it was larger for each combination of set size and range. We then averaged these simulations, so the values in Tables 1 and 2 are based on 40,000 simulated trials for each cell. For each trial, we calculated whether or not sampling one, two, three, or four items and judging whether the probe was bigger or smaller than the mean based on the average of the sampled items would produce a correct response.

With the smaller range of stimuli, separated by a factor of $5 \%$ on a log scale, sampling just one item from the array produced performance comparable to that found by Ariely (2001), $\sim 75 \%$ correct performance for size differences of $6 \%$ or greater. Moreover, as in the original study, the number of objects in the array had no effect on performance. For all set sizes, sampling one item was sufficient to produce $\sim 75 \%$ correct performance.

In contrast, the sampling strategy was not effective for the larger range of stimuli used in the original study
(Ariely, 2001). With a greater separation between items, increasing the probe size by a smaller percentage than the difference between items meant that the individual items in the array did not discriminate between the probe and the actual mean. In fact, for set sizes larger than four, even randomly sampling four items and averaging those is relatively ineffective with this larger stimulus range. Not surprisingly, our replication using a $10 \%$ increment between item sizes produced performance intermediate to the smaller and larger ranges used by Ariely.

Although the random sampling strategy was ineffective with the larger stimulus range, subjects could still perform the task without averaging all of the items; other sampling strategies are effective. For example, finding and then averaging just the largest and smallest items in the array produces effectively perfect performance for size differences of $5 \%$ or greater. Accurate performance does not require averaging of all the items in the set. And in debriefing during our experiments, many subjects anecdotally reported using

Table 2

Simulation of the Effectiveness of Using a Sampling Strategy

With Stimuli Approximating Those Using a Larger Scaling Factor From Ariely (2001)

\begin{tabular}{|c|c|c|c|c|c|c|c|c|c|c|c|c|c|c|c|c|}
\hline \multirow{2}{*}{$\begin{array}{c}\text { Difference } \\
(\%)\end{array}$} & \multicolumn{4}{|c|}{ No. Sampled From 4 Objects } & \multicolumn{4}{|c|}{ No. Sampled From 8 Objects } & \multicolumn{4}{|c|}{ No. Sampled From 12 Objects } & \multicolumn{4}{|c|}{ No. Sampled From 16 Objects } \\
\hline & 1 & 2 & 3 & 4 & 1 & 2 & 3 & 4 & 1 & 2 & 3 & 4 & 1 & 2 & 3 & 4 \\
\hline 16 & .5003 & & & & & & & & & & & 31 & & & .5975 & .6150 \\
\hline & & & & & & & & & & & & & & & & \\
\hline & & & & & & & & & & & & & & & & \\
\hline 1 & & & & & & & & & & & & & & & & \\
\hline 1 & & .66 & & & & & & & & & & & & & & 09 \\
\hline 11 & & .501 & & & & & & & & & & & & & & .5758 \\
\hline 10 & & .5016 & & & & & & & & & & & & & & .5758 \\
\hline & & & & & & & & & & & & & & & & \\
\hline & & & & & & & & & & & & & & & & .5710 \\
\hline & & & & & & & & & & & & & & & & .57 \\
\hline 6 & & & & & & & & & & & & & & & & .5665 \\
\hline 5 & .5003 & .5016 & .5018 & & .5041 & & .49 & & & .49 & & & .4994 & & & .5665 \\
\hline 4 & & .5016 & & & & & & & & & & & & & & .5626 \\
\hline & & & & & & & & & & & & & & & & .5673 \\
\hline 2 & & & & & & & & & & & & & & & & .5673 \\
\hline 1 & .5003 & .5016 & .5018 & 1.0000 & .5041 & .5001 & .4974 & .6129 & .4969 & .4994 & .5000 & .5790 & .4994 & .5026 & .4984 & .5673 \\
\hline
\end{tabular}


such a strategy. Whether or not subjects actually use such a strategy is an open question, but evidence that they could perform the task accurately by doing so weakens claims that they must be using a new averaging mechanism.

\section{OTHER EVIDENCE FOR AND AGAINST AVERAGING}

Although our results and simulations, as well as the experiments in de Fockert and Marchant (2008), are consistent with our focused-attention alternative to the perception of average size, such strategies might prove effective only with the array/probe method of studying average size perception. A number of other experiments have examined the nature and limits of perceptual size averaging, using methods other than those in Ariely (2001).

Below, we describe these alternative methods and report simulations of many of the published findings, to test whether alternative focused-attention strategies that do not exceed established limits on focused attention and visual working memory can effectively account for actual performance. Table 3 provides a synopsis of the results of all of these simulations and notes whether the results of the original experiments can be explained by various focusedattention strategies.

\section{Two-Array Comparisons}

Other than the Ariely (2001) method of presenting an array followed by a single probe, the most common task used to study average size perception requires subjects to judge which of two arrays has a larger average (Chong \& Treisman, 2003, 2005b). An advantage of the task of comparing two arrays, rather than comparing one array with a test probe, is that it is easier to determine whether people treat different distributions of sizes in similar ways. More important, it makes a strategy of random selection less effective because of the added variance. In the single-item probe task, all of the variability in the performance of the simulation comes from the variability within the one array. In the two-array case, each selection adds some variability to the judgment.

A disadvantage of the two-array method is that the manipulation used to introduce a difference in the means of the two arrays can introduce other ways to perform the task that do not require perceiving the mean size. For example, in many studies in which the two-array method has been used, the item sizes in one array have been multiplied by a constant factor to produce a second array with a mean that is bigger/smaller. However, if all of the objects in one array are bigger than the corresponding objects in the other array by a constant multiplier, observers can correctly determine which array has the larger mean simply by finding the largest (or smallest) item in the entire display; the largest item will always come from the side with the larger mean size, and the smallest item will always come from the array with the smaller mean size. This simple strategy would work for many of the published experiments in which the two-array method was used (see Table 3).

Other experiments have eliminated the find the largest strategy and still have obtained reasonably good performance (see Chong \& Treisman, 2003). However, manipulations that affect the relative sizes of the means of two arrays also affect other aspects of the array, which can then allow simpler focused-attention strategies to become effective. In some cases, these manipulations allow the random sampling strategy to produce good performance. In others, averaging the biggest and largest items in each array produces good performance, just as it does in the array/probe method. Below, we provide simulations for each of these methods and tasks.

\section{Simulation 2: Two Arrays With Uniform Distributions (Chong \& Treisman, 2003)}

Most of the experiments using the two-array approach have been reported by Chong and Treisman $(2003,2005 b)$. The 2003 article focused on average size perception when

Table 3

Effectiveness of Various Focused Attention Strategies in Explaining Existing Evidence for Average Size Perception

\begin{tabular}{|c|c|c|c|c|c|c|}
\hline \multirow[b]{2}{*}{ Study } & \multirow[b]{2}{*}{ Finding } & \multicolumn{5}{|c|}{ Strategies } \\
\hline & & Sample 1 & Sample 2 & Sample 3 & $\begin{array}{l}\text { Find Largest } \\
\text { (Smallest) }\end{array}$ & $\begin{array}{c}\text { Mean } \\
\text { (Largest, Smallest) }\end{array}$ \\
\hline \multirow[t]{2}{*}{ Ariely (2001) } & Small range of sizes & + & + & + & - & + \\
\hline & Large range of sizes & - & - & - & - & + \\
\hline \multirow{4}{*}{ C\&T (2003) } & Experiment 1 & - & - & - & + & \\
\hline & Experiment 2 & - & - & - & + & \\
\hline & Experiment 3 nonhomogenous & - & - & - & + & \\
\hline & Experiment 3 homogenous vs. other & - & $+1-$ & $+1-$ & - & + \\
\hline $\mathrm{C} \& \mathrm{~T}(2005 \mathrm{~b})$ & $\begin{array}{l}\text { Experiment } 1 \text { with varied proportions } \\
\text { of the same sizes }\end{array}$ & - & + & + & - & $\begin{array}{l}+ \text { (with comparison } \\
\text { to more frequent item } \\
\text { in other array) }\end{array}$ \\
\hline C\&T (2005a) & Array + forced choice between probes & + & + & + & - & \\
\hline C\&T (2005a) & $\begin{array}{l}\text { Varied proportions with array }+ \text { forced choice } \\
\text { between probes }\end{array}$ & $+1-$ & + & + & & \\
\hline
\end{tabular}

Note_- "+ "means that the strategy produces simulated performance comparable to that of actual subjects; "- " means that the strategy cannot explain performance; "+/-" means that the strategy could work in some conditions; and no symbol means that the possibility could not be determined, was not tested, or was not applicable for that method. "C\&T" refers to Chong and Treisman. The table reflects only the ability to account for the overall level of performance and does not reflect other aspects of performance (e.g., differences between member identification and average size perception, set size effects, etc.). The simulated data typically are consistent with these effects as well (see the text). For studies using two arrays, the sampling strategies are based on sampling that many items from each side (e.g., "Sample 2" means sampling two items from each array, for a total of four items), except for homogenous arrays for which all items are identical. 
Table 4

Simulation of Sampling Strategies for Experiment 1 From Chong and Treisman (2003)

\begin{tabular}{lcccccc}
\hline & \multicolumn{5}{c}{ Difference (\%) Between Arrays } \\
\cline { 2 - 7 } Per Side & 2 & 4 & 6 & 8 & 10 & 12 \\
\hline Sample one & .6296 & .6261 & .6300 & .6280 & .6264 & .6263 \\
Sample two & .5650 & .5734 & .6022 & .6604 & .6564 & .6760 \\
Sample three & .5572 & .5857 & .6646 & .7020 & .6990 & .7294 \\
Sample four & .5518 & .5834 & .6802 & .7026 & .7052 & .7584 \\
\hline
\end{tabular}

Note-Values in the table represent the proportions correct under various sampling assumptions. In the study and simulation, each array had 12 items at four different sizes ( 3 items per size). The base array in our simulation consisted of circles with the following diameters (in degrees of visual angle): $1.82,1.82,1.82,2.27,2.27,2.27,2.84,2.84,2.84,3.55$, 3.55 , and 3.55 . For the comparison array, each element was incremented by $2 \%, 4 \%, 6 \%, 8 \%, 10 \%$, or $12 \%$. For each combination of size difference, we simulated 20,000 trials and calculated the average performance for randomly sampling 1, 2, 3, and 4 items from each array.

the two arrays had different distributions, although the first two experiments used uniform distributions for both arrays. We first simulated performance with uniform arrays that were the same as those used by Chong and Treisman (2003), to determine whether the strategy of selecting subsets of items at random from each side of the display would prove sufficient to produce performance comparable to that of their subjects. Our simulation (see Table 4) assumed that the observer sampled some subset of items from each array, calculated the mean for each subset, and compared the two means with perfect precision to decide which side had the larger mean. The actual subjects in these studies correctly chose the larger array on $75 \%$ of the trials, with a size differences of $8 \%$ between the means of the two arrays.

The simulation showed that subjects would need to sample at least four items from each array (a total of eight or more items) to achieve $75 \%$ correct performance, and even then, they would need a $12 \%$ difference between the arrays to achieve that level of performance. Not surprisingly, a simulation of Experiment 2 from Chong and Treisman (2003) produced nearly identical results (see Table 5). Thus, the strategy of selecting a random subset of items from each array does not sufficiently explain performance with this variant of the two-array task. (Note, though, that selecting just one item per array produces reasonably good performance, with $>60 \%$ accuracy even for a $2 \%$ size difference.)

Table 5

Simulation of Sampling Strategies for Experiment 2 From Chong and Treisman (2003)

\begin{tabular}{lcccccc}
\hline & \multicolumn{5}{c}{ Difference (\%) Between Arrays } \\
\cline { 2 - 7 } Per Side & 2 & 4 & 6 & 8 & 10 & 12 \\
\hline Sample one & .6228 & .6207 & .6236 & .6269 & .6306 & .6236 \\
Sample two & .5587 & .5700 & .5938 & .6559 & .6571 & .6692 \\
Sample three & .5542 & .5766 & .6639 & .6978 & .6988 & .7193 \\
Sample four & .5491 & .5780 & .6744 & .7036 & .7044 & .7484 \\
\hline
\end{tabular}

Note-Values in the table represent the proportions correct under various sampling assumptions. The simulation details were the same as those in Table 4, except that the base array of circles had the following diameters (in degrees of visual angle): 1.63, 1.63, 1.63, 2.04, 2.04, 2.04, 2.55, $2.55,2.55,3.18,3.18$, and 3.18 .
Although the strategy of selecting a subset of items at random from each array and comparing the means of those subsets does not adequately account for performance in this task, other strategies would work effectively. Given the method for generating the size difference between the arrays, performance of an ideal observer would be perfect simply by finding the biggest (or smallest) item in the display. Actual observers are not ideal, though, and even when comparing individual items (in Experiment 1), they did not achieve perfect performance. And, on the basis of Figure 1 in Chong and Treisman (2003), performance in the simultaneous condition was comparable for single items and arrays, which is consistent with the idea that the subjects tried to find the largest item in the display but that their sensitivity to small differences led to some errors in finding the biggest item. In summary, the strategy of randomly selecting subsets of items from each array proved ineffective with this task, but a strategy of finding the biggest (or smallest) item in the entire array would produce the correct results without any need for averaging. Consequently, these experiments do not provide direct support for a global, parallel average size perception mechanism, and they do not require averaging of more than three or four items.

\section{Simulation 3: Two Arrays With Varied \\ Distributions (Chong \& Treisman, 2003)}

Experiment 3 in the same article (Chong \& Treisman, 2003) was designed to eliminate the strategy of finding the largest (or smallest) item in the array by using different distributions of sizes in the two arrays. The study compared performance when the two arrays had one of several distributions: uniform (equal frequencies of each size, with equal spacing between sizes), bimodal (half the objects were the largest size and half were the smallest), homogenous (all circles of the same size), and normal (four of each of the middle sizes and two each at the largest and smallest sizes). Although the goal of this experiment was to eliminate the strategy of finding the largest or the smallest item in the array, most of the conditions could be solved with this strategy. In fact, all comparisons among the uniform, normal, and bimodal distributions are subject to the strategy of finding the largest or the smallest item in the display; in all of these cases, the largest item in the display is on the side with the larger mean size.

Before examining the conditions that are not subject to this strategy, we first verified that, like the comparison of two uniform distributions in Simulation 2, comparisons of uniform and bimodal distributions could not be performed successfully by sampling a small subset of items from each side (see Table 6). Although simulated performance with these displays is slightly better for smaller size differences than it was when comparing two uniform distributions (Simulation 2), simulated performance still was worse than actual performance, and the strategy of random sampling from each side would need to exceed the known limits of focused attention to achieve levels of performance comparable to those of actual subjects. 
Table 6

Simulation of Sampling Strategies

for Comparison of a Uniform With a Bimodal Array

From Experiment 3 in Chong and Treisman (2003)

\begin{tabular}{lcccccc}
\hline & \multicolumn{5}{c}{ Difference (\%) Between Arrays } \\
\cline { 2 - 7 } Per Side & 2 & 4 & 6 & 8 & 10 & 12 \\
\hline Sample one & .6235 & .6262 & .6233 & .6274 & .6236 & .6198 \\
Sample two & .5826 & .5910 & .6076 & .6836 & .6824 & .6842 \\
Sample three & .5720 & .6030 & .6844 & .6864 & .6855 & .7214 \\
Sample four & .6186 & .6451 & .7233 & .7350 & .7330 & .7666
\end{tabular}

Note-Values in the table represent the proportions correct under various sampling assumptions.

One of the comparisons that does not permit a strategy of finding the biggest item in the array is the comparison of a distributed array of item sizes with a homogenous array in which all of the items have the same size. Note, though, that this condition reduces to the single-probe method in which the mean of an array is compared with the single-item size (Ariely, 2001). And, as is shown in our simulations and data, depending on the relative sizes of the items in the array, such a task can be performed either by selecting just one or two items from the array or by averaging the biggest and smallest items in the array. Given that the size range used by Chong and Treisman (2003) was different from that used by Ariely, we simulated the homogenous versus uniform and the homogenous versus bimodal conditions in Chong and Treisman (2003), as is shown in Tables 7 and 8 .

For the homogenous versus uniform comparison, performance reached $\sim 75 \%$ with an $8 \%$ size difference by sampling three circles from the uniform distribution and comparing the mean of those with any item from the homogenous side. Given that this sampling strategy is

Table 7

Simulation of Sampling Strategies

for Comparison of a Homogenous With a Uniform Array From Experiment 3 in Chong and Treisman (2003)

\begin{tabular}{lcccccc}
\hline & \multicolumn{6}{c}{ Difference (\%) Between Arrays } \\
\cline { 2 - 7 } Per Side & 2 & 4 & 6 & 8 & 10 & 12 \\
\hline Sample one & .5010 & .4989 & .4977 & .4984 & .5043 & .5022 \\
Sample two & .5032 & .6366 & .6428 & .6320 & .6456 & .6391 \\
Sample three & .5044 & .6236 & .7084 & .7435 & .7528 & .7456 \\
Sample four & .5874 & .6054 & .7706 & .7748 & .7800 & .8314 \\
\hline
\end{tabular}

Note-Values in the table represent the proportions correct under various sampling assumptions.

Table 8

Simulation of Sampling Strategies for Comparison of a Homogenous With a Bimodal Array From Experiment 3 in Chong and Treisman (2003)

\begin{tabular}{lcccccc}
\hline & \multicolumn{6}{c}{ Difference (\%) Between Arrays } \\
\cline { 2 - 7 } Per Side & 2 & 4 & 6 & 8 & 10 & 12 \\
\hline Sample one & .4946 & .5043 & .5070 & .4996 & .5052 & .5011 \\
Sample two & .7721 & .7738 & .7734 & .7717 & .7769 & .7714 \\
Sample three & .4976 & .5018 & .5054 & .9062 & .9084 & .9090 \\
Sample four & .7297 & .7274 & .7308 & .7269 & .7258 & .7246 \\
\hline
\end{tabular}

Note-Values in the table represent the proportions correct under various sampling assumptions. within the estimated four-item capacity of focused attention, selecting a subset would appear to be a possible strategy for this condition, although it might be more difficult than finding the biggest and smallest items in the array and averaging those, which would also produce excellent performance.

For the homogenous versus bimodal comparison, sampling an even number of items from the bimodal display consistently produced $75 \%$ performance regardless of the size of the difference. Sampling one item produced $50 \%$ performance. Sampling three from each side gave $50 \%$ performance for small differences and $90 \%$ performance for big differences. Thus, performance with this pairing of nonuniform distributions could be solved with focused attention by selecting any two items at random from the bimodal side of the display. Of course, it could also be solved even more accurately by averaging one of the big items and one of the small items (equivalent to the biggest and smallest).

In summary, the only conditions in Experiment 3 from Chong and Treisman (2003) that are problematic for the strategy of finding the largest item in the array are those involving the homogenous condition in which all of the items on one side are the same as the mean for that side. Note that these comparisons reduce to the Ariely (2001) method; it is equivalent to comparing a test array with a single probe item. Such comparisons can be solved successfully by sampling fewer than four items from the nonhomogenous side or by averaging the biggest and smallest items on the nonhomogenous side. In all cases, subjects could adopt a relatively simple focused-attention strategy that would accomplish the task without needing to average all of the items in the display.

\section{Simulation 4: Two Arrays With Varied Proportions of Each Size (Chong \& Treisman, 2005b)}

Another approach that can eliminate the strategy of picking just one item from a side (e.g., the biggest item) is to vary the proportion of objects of each size in the array. By varying the proportions, no one object in either array can determine the correct answer. The critical question, though, is whether performance with varied proportions of items requires averaging of all the items in the display (or at least more than the capacity of attention) or whether performance is also consistent with sampling a small number of items. Here, we simulated one such experiment that used the two-array approach (Experiment 1 from Chong \& Treisman, 2005b). Each array used only two sizes of objects, but the actual sizes used and the relative proportions of the two sizes was varied. One array had objects of two different sizes (diameters of $1.36^{\circ}$ and $1.66^{\circ}$ of visual angle) in varying proportions (six of one and two of the other, three of one and five of the other). The other array also had only two sizes of objects but had four of each. Size differences of $7 \%, 13 \%,-7 \%$, and $-13 \%$ were created by changing the size of the items in the equally distributed side. We used sizes of objects that were the same as those used in the original experiment. However, we simulated only the condition with 8 objects in each array, 
Table 9

Simulation of Experiment 1 From Chong and Treisman (2005b) Comparing Arrays With Different Proportions of Objects of Each Size

\begin{tabular}{|c|c|c|c|c|c|c|}
\hline \multirow[b]{2}{*}{ Array 1} & \multirow[b]{2}{*}{ Array 2} & \multirow{2}{*}{$\begin{array}{c}\text { Difference } \\
(\%)\end{array}$} & \multicolumn{4}{|c|}{ Number Sampled } \\
\hline & & & 1 & 2 & 3 & 4 \\
\hline $1.36(n=2), 1.66(n=6)$ & $1.1626(n=4), 1.7724(n=4)$ & 7 & .5038 & .7646 & .7200 & .7662 \\
\hline $1.36(n=3), 1.66(n=5)$ & $1.0350(n=4), 1.6714(n=4)$ & 13 & .4949 & .7236 & .8875 & .9124 \\
\hline $1.36(n=5), 1.66(n=3)$ & $1.2703(n=4), 1.8599(n=4)$ & -7 & .4962 & .7249 & .8687 & .7907 \\
\hline $1.36(n=6), 1.66(n=2)$ & $1.3210(n=4), 1.9017(n=4)$ & -13 & .5008 & .7684 & .9284 & .9808 \\
\hline
\end{tabular}

Note-For each simulation, we randomly selected samples of various numbers of items from each array, computed the mean of these perceptual areas, converted back to raw diameter, and then compared them. For each of the 20,000 samples/condition, we scored it as correct if it produced the same result as the comparison of the array means. We simulated the four pairings of eight-item array types used in the experiment.

on the assumption that the condition with 16 in each array would produce similar results (see Simulation 1 above; also, a preliminary simulation comparing displays with 8 items in one array and 16 in the other appears to produce similar results). We also did not simulate the effects of density, since they are tangential to the purpose of our article. Finally, we did not simulate Experiment 2 given the added complexity of the wide range of size differences used. Given our simulated results for a $7 \%$ size difference (see Table 9), simulating the wider range of sizes seemed unnecessary.

For all the conditions, sampling just one item from each side produced chance performance (as would be expected given that the experiment was designed to eliminate this strategy). However, sampling two items from each side produced $\sim 75 \%$ performance. Thus, subjects could perform this task by sampling up to two items from each side and making their judgment on the basis of that subset. We did not simulate sampling one item from one side and two from the other, but that presumably would produce intermediate performance. Even though the distributions were changed to make sampling of one item from each side ineffective as a strategy, the resulting proportioned distributions lend themselves to a strategy of selecting two from each side, meaning that performance at the level shown by the subjects could, in principle, be obtained by sampling four or fewer items in the display with focused attention. Another strategy that proves effective with these displays is to average one item of each size from the equally distributed array and compare that with the more frequent size in the other array. For example, for comparisons like that in row 1 of Table 9, take the average of 1.1626 and 1.7724 and compare that with 1.66 (the more frequent item in the other array). This strategy would produce perfect performance without a need to average all the items in the array. We do not claim that people do use such strategies. Rather, we claim that they could, meaning that evidence for good performance in this task does not require averaging of all of the items in each array; in principle, it could be accomplished within the limits of focused attention.

\section{Simulation 5: One Array Followed by a Forced Choice Between Two Probes (Chong \& Treisman, 2005a)}

Another task used to explore average size perception modified the original Ariely (2001) method by presenting an array followed by two test probes. Observers judged which of the two probes was the same size as the mean of the array elements. In the only published experiments using this method (Chong \& Treisman, 2005a), a staircase technique was employed to adjust the difference in size between the two test probes across trials until observers achieved $80 \%$ accuracy in selecting the probe that matched the average size in the array. Somewhat surprisingly, performance with this forced choice task was substantially worse than that in any of the experiments discussed thus far, including our replications (although it was closer to performance in our Experiment 2). With this adjustment method, more than a $20 \%$ size difference was needed for $80 \%$ accuracy in almost all conditions. In previous studies, subjects achieved $75 \%$ performance with probes that differed from the mean of the array by as little as $4 \%-12 \%$. Given the hypothesis that subjects can perceive the average size of an array by using a global, parallel averaging mechanism and that they use this summary representation in their judgments, it is unclear why performance should vary so substantially across what appear to be similar methods. In contrast, if subjects are not perceiving the average but, instead, are using various focused-attention strategies, it is conceivable that performance would vary more as a function of the particular strategy used for that task.

We simulated the results of one experiment in which this forced choice method was used (Experiment 1 from Chong \& Treisman, 2005a). The experiment was designed to examine the effects of focused- versus diffuse-attention tasks on judgments of the mean size and on recognition of individual elements of the array. Below, we address the implications of that manipulation, but first we will present simulation data to determine whether averaging was necessary to perform the task. We simulated performance for arrays of 4 unique items and arrays with 12 items (3 at each size), using the same sizes as those in the original experiment (see Tables 10 and 11).

By sampling just one item from the array, subjects could achieve $\sim 75 \%$ correct performance in both conditions. By sampling two items, they would exceed $80 \%$ correct, with a difference of $17 \%$ in both conditions as well. Thus, performance in this task requires that subjects sample only one or two items to achieve performance comparable to that found in the experiment. Given that one or two items is fewer than the three- or four-item capacity of focused attention, accu- 
Table 10

Simulation of Sampling Strategies for Four-Item Displays in Experiment 1 From Chong and Treisman (2005a)

\begin{tabular}{cccccc}
\hline $\begin{array}{c}\text { Size Difference } \\
\text { Between Probes }\end{array}$ & Foil Probe & & \multicolumn{4}{c}{ Number Sampled } \\
\cline { 3 - 6 }$(\%)$ & Diameter & 1 & 2 & 3 & 4 \\
\hline 50 & 4.5398 & .7436 & 1.0000 & 1.0000 & 1.000 \\
47 & 4.4903 & .7436 & 1.0000 & 1.0000 & 1.000 \\
44 & 4.3582 & .7436 & 1.0000 & 1.0000 & 1.000 \\
41 & 4.2674 & .7436 & 1.0000 & 1.0000 & 1.000 \\
38 & 4.1766 & .7436 & 1.0000 & 1.0000 & 1.000 \\
35 & 4.0858 & .7436 & 1.0000 & 1.0000 & 1.000 \\
32 & 3.9950 & .7436 & 1.0000 & 1.0000 & 1.000 \\
29 & 3.9042 & .7436 & .8332 & 1.0000 & 1.000 \\
26 & 3.8134 & .7436 & .8332 & 1.0000 & 1.000 \\
23 & 3.7226 & .7436 & .8332 & 1.0000 & 1.000 \\
20 & 3.6318 & .7436 & .8332 & 1.0000 & 1.000 \\
17 & 3.5411 & .7436 & .8332 & 1.0000 & 1.000 \\
14 & 3.4503 & .7436 & .6614 & 1.0000 & 1.000 \\
11 & 3.3595 & .7436 & .6614 & .7506 & 1.000 \\
8 & 3.1779 & .4974 & .6614 & .7506 & 1.000 \\
5 & 3.2687 & .4974 & .6614 & .5038 & 1.000 \\
2 & 3.0871 & .4974 & .6614 & .5038 & 1.000 \\
\hline
\end{tabular}

Note-In the study and simulation, each array had four items at four different sizes. The array consisted of circles with a mean of 3.0266 and the following diameters (in degrees of visual angle): 2.4, 2.7367, 3.1829, 3.7866 (numbers were calculated from the smallest size of 2.4 listed in the article coupled with a $1.15 \times$ increase for each item on a log scale. Given that the staircase procedure used $3 \%$ increments or decrements in size, we simulated performance with size differences ranging from $50 \%$ to $2 \%$ in $3 \%$ increments. We generated 20,000 trials and sampled one, two, three, or four items at random (without replacement) on each trial. For each sample, we determined whether the sampled item was closer to the actual mean or to the other probe in the forced choice, given that size difference. For simplicity, the foil probes were always larger than the actual mean but given that the items were uniformly distributed, the results should be identical if the percentage difference made the foil smaller. Numbers in the figure constitute the proportion correct out of 20,000 trials for a given sample size and the percentage difference between the mean and the probe. The table also shows the foil probe diameter for comparison.

rately choosing the correct probe in this task could be accomplished with focused attention and without a need to average all (or even most) of the items in the array. Although we did not simulate the other experiments in Chong and Treisman (2005a), the basic result should be comparable.

In addition to using a modified version of the Ariely (2001) method to examine averaging performance, Chong and Treisman (2005a) also examined whether judging the mean size of an array of objects would be affected by whether attention was focused narrowly or broadly. The experiment manipulated attention in several ways, including using a serial or parallel search task and using a rectangle judgment task in which the rectangle was located at fixation or surrounding the entire array. If perceiving the average size of an array of objects depends on parallel processing of the entire array or distributed attention across the array, performance should be better when attention is distributed. In contrast, memory for individual items in the array should be better with focused attention to each item. The experiments showed exactly that: Performance in the mean judgment task was relatively better with the distributed-attention task than with the focusedattention task, with the opposite pattern for recognition of individual elements (as revealed through a significant interaction term, although the differences within a condition were not significant in Experiment 1). This finding suggests distinct mechanisms for perceiving the mean size of the elements of an array and perceiving the individual elements, and on its surface, it seems inconsistent with our alternative explanation that the subjects used focused attention to do the mean judgment task.

Note, though, that our simulation shows that averaging is not needed for the task and that subjects need to encode only one or two items from the array to achieve performance comparable to that found in the experiment. Although the effect of focused versus distributed attention does imply distinct processes in the mean judgment task and the member recognition task, the interaction might result from the different demands of these two tasks on memory and attention, and not from the use of a distinct, distributed averaging process. That is, the interaction may reflect the differential demands of trying to remember as many individual items as possible for a recognition test and trying to remember the average of one or two items for a mean judgment task. More specifically, when the search task is hard, it interferes with the ability to quickly sample one or two items, perhaps due to proactive or retroactive interference during the search. In the easy task, subjects can find the target and sample it (and possibly, one surrounding item) without much challenge, because the search itself is easy and does not produce interference from focusing on additional items. In contrast, for the member judgment task, the goal is to remember as many of the items as possible in the time permitted, and focusing individually on each item should aid memory performance for those items; the hard search takes longer and is more consistent with the judgment they will have to make, whereas the easy search gives less time to focus on many items.

Note that this account does not require a distributed averaging process. The result just reflects the dissimilarity

Table 11

Simulation of Sampling Strategies for 12-Item Displays in Experiment 1 From Chong and Treisman (2005a)

\begin{tabular}{cccccc}
\hline Size Difference & & \multicolumn{4}{c}{ Number Sampled } \\
\cline { 3 - 6 } $\begin{array}{c}\text { Between Probes } \\
(\%)\end{array}$ & Foil Probe & & \multicolumn{4}{c}{ Diameter } & 1 & 2 & 3 & 4 \\
\hline 50 & 4.5398 & .7458 & .9542 & .9948 & 1.0000 \\
47 & 4.4903 & .7458 & .9542 & .9948 & 1.0000 \\
44 & 4.3582 & .7458 & .9542 & .9948 & 1.0000 \\
41 & 4.2674 & .7458 & .9542 & .9948 & 1.0000 \\
38 & 4.1766 & .7458 & .9542 & .9948 & .9923 \\
35 & 4.0858 & .7458 & .9542 & .9518 & .9923 \\
32 & 3.9950 & .7458 & .9542 & .9518 & .9860 \\
29 & 3.9042 & .7458 & .8174 & .9518 & .9673 \\
26 & 3.8134 & .7458 & .8174 & .9118 & .9614 \\
23 & 3.7226 & .7458 & .8174 & .8704 & .9614 \\
20 & 3.6318 & .7458 & .8174 & .8704 & .8994 \\
17 & 3.5411 & .7458 & .8174 & .8315 & .8452 \\
14 & 3.4503 & .7458 & .6794 & .8315 & .8266 \\
11 & 3.3595 & .7458 & .6794 & .7084 & .7702 \\
8 & 3.1779 & .4998 & .6342 & .7034 & .7156 \\
5 & 3.2687 & .4998 & .6342 & .5806 & .6082 \\
2 & 3.0871 & .4998 & .4964 & .5381 & .5836 \\
\hline
\end{tabular}

Note-The simulation was identical to the one in Table 10, except that three items at each size were included in the array. 
of the task demands of the two types of search and the two types of judgments. The same sort of argument applies to the rectangle judgment task (which examined only mean judgments and not member judgments); requiring subjects to focus narrowly at the center of the screen might impair their ability to quickly sample one or two items (because attention is zoomed in). Focusing attention on a rectangle surrounding the item might make it easier to sample one or two items. The lack of a set size effect in these experiments would not be surprising if the subjects used only one or two items from the array to make their decision.

\section{Simulation 6: One Array With Forced Choice Between Two Probes, With Varied Proportions of Two Sizes (Experiment 4 from Chong \& Treisman, 2005a)}

Simulation 5 showed that subjects can perform a forced choice between two test probes fairly accurately by sampling only one or two items from the array. Experiment 4 in Chong and Treisman's (2005a) article was intended to avoid this problem: "By varying the frequencies of each size, we ensured that the whole set would have to be processed to ensure accurate judgments" (p. 10). In their experiment, the subjects needed a difference between the probes of $20 \%-27 \%$, depending on the condition, in order to achieve $80 \%$ accuracy. Here, we simulated performance with a sampling strategy to determine whether this manipulation requires processing of the entire set in order to achieve the obtained level of accuracy.
For our simulation, we used the same combinations of the two diameters $\left(1.1^{\circ}\right.$ and $\left.1.7^{\circ}\right)$ tested in the study. Specifically, we simulated conditions with four of each size, three of one size and five of the other, and six of one size and two of the other. We conducted separate simulations for probes that were larger than the mean and probes that were smaller than the mean and then combined the results in Table 12. For simplicity, we also averaged across which size had more or fewer items present (e.g., we averaged across the $5 / 3$ and $3 / 5$ conditions). Finally, although we also simulated performance for sampling three and four items, we will report only the results for sampling one or two items, because that proved sufficient for all the conditions.

Averaging across all the conditions (as in Chong \& Treisman, 2005a, Experiment 4), simulated performance exceeded $80 \%$ correct by sampling two items from the array at random, with only a $26 \%$ difference between the probes. Thus, the subjects did not need to process the entire set in order to achieve an accurate judgment of the mean size of the array. In many conditions, other strategies would work as well. For example, in some cases, the foil probe was either larger than the largest array item or smaller than the smallest array item, meaning it could be dismissed if the subjects had perceived the largest and smallest items in the set. Again, that does not require subjects to perceive the average, and overall performance might be boosted by such strategies beyond the relatively good performance that could be achieved by randomly sampling just two items.

Table 12

Simulation of the Effectiveness of a Sampling Strategy in Accounting for Experiment 4 From Chong and Treisman (2005a)

\begin{tabular}{|c|c|c|c|c|c|c|c|c|}
\hline \multirow{3}{*}{$\begin{array}{c}\text { Size Difference } \\
(\%)\end{array}$} & \multicolumn{8}{|c|}{ Condition } \\
\hline & \multicolumn{2}{|c|}{$\begin{array}{l}\text { 4/4 Number } \\
\text { Sampled }\end{array}$} & \multicolumn{2}{|c|}{$\begin{array}{l}\text { 3/5 Number } \\
\text { Sampled }\end{array}$} & \multicolumn{2}{|c|}{$\begin{array}{c}\text { 6/2 Number } \\
\text { Sampled }\end{array}$} & \multicolumn{2}{|c|}{$\begin{array}{l}\text { All Conditions } \\
\text { Number Sampled }\end{array}$} \\
\hline & 1 & 2 & 1 & 2 & 1 & 2 & 1 & 2 \\
\hline 50 & 1.0000 & 1.0000 & .8178 & .9363 & .8756 & .9826 & .8774 & .9676 \\
\hline 47 & 1.0000 & 1.0000 & .8206 & .9390 & .8756 & .9826 & .8784 & .9687 \\
\hline 44 & 1.0000 & 1.0000 & .8234 & .9180 & .8756 & .9826 & .8796 & .9698 \\
\hline 41 & .5003 & .7842 & .8261 & .9446 & .8756 & .9826 & .7808 & .9277 \\
\hline 38 & .5003 & .7842 & .8289 & .9473 & .8756 & .9826 & .7819 & .9288 \\
\hline 35 & .5003 & .7842 & .8317 & .9501 & .8756 & .9826 & .7830 & .9299 \\
\hline 32 & .5003 & .7842 & .6944 & .8757 & .8756 & .9826 & .7280 & .9002 \\
\hline 29 & .5003 & .7842 & .5295 & .7864 & .8756 & .9826 & .6613 & .8644 \\
\hline 26 & .5003 & .7842 & .5423 & .7891 & .8756 & .9826 & .6672 & .8655 \\
\hline 23 & .5003 & .7842 & .5450 & .7919 & 6887 & .7419 & .5936 & .7704 \\
\hline 20 & .5003 & .7842 & .5478 & .7946 & 6887 & .7419 & .5947 & .7715 \\
\hline 17 & .5003 & .7842 & .5506 & .7974 & .4998 & .4968 & .5202 & .6745 \\
\hline 14 & .5003 & .7842 & .5534 & .8002 & .4998 & .4968 & .5213 & .6756 \\
\hline 11 & .5003 & .7842 & .5561 & .6696 & .4998 & .4968 & .5224 & .6234 \\
\hline 8 & .5003 & .7842 & .5589 & .5715 & .4998 & .4968 & .5235 & .5842 \\
\hline 5 & .5003 & .7842 & .5617 & .5743 & .4998 & .4968 & .5246 & .5853 \\
\hline 2 & .5003 & .7842 & .5644 & .5433 & .4998 & .4968 & .5258 & .5729 \\
\hline
\end{tabular}

Note-Each cell in the 4/4 condition is based on 40,000 trials, 20,000 with each probe size (bigger or smaller than the mean of the array). Each cell in the other conditions is based on 80,000 simulated trials that averaged across both the size of the probe and which item had each proportion (e.g., three items or five items). Each cell in the "All Conditions" column averages the other cells, weighting for the proportion of trials in the actual experiment. The original experiment did not report the step size used in the staircase procedure, so we used a $3 \%$ increment in our simulations. Our simulation also assumed that the size difference between the two probes was generated relative to the actual mean of the array (which varies depending on the proportions of items in the array). That is, one probe had the mean size of the array, and the other was incremented or decremented by some percentage. 


\section{GENERAL DISCUSSION}

The results from our experiments and simulations show that judgments of the average size of a set of items do not require a global, parallel averaging mechanism. The data from all the published methods used to study average size perception are consistent with focused-attention strategies that do not exceed the accepted capacity limits of focused attention. Judgments of whether a probe stimulus is bigger or smaller than the mean of a set of items could be accomplished by sampling just one or two items from the set or by averaging the biggest and smallest items in the set. Comparisons of two separate sets to determine which has a larger mean can be accomplished by finding the largest item in the display in some cases or by sampling a small number of items from each side in other cases. Judgments of which of two probes match the mean of a set of items could be accomplished by sampling just one or two items from the set. We have found no published cases for which performance exceeds the limits of focused attention and no cases for which averaging of all of the items in the set is necessary to explain task performance.

Several other forms of evidence have been offered to support the posited global, parallel averaging mechanism: (1) Performance on averaging is better with distributed than with focused attention (Chong \& Treisman, 2005a); (2) performance on average size judgments exceeds recognition memory for individual items in the set (Ariely, 2001); and (3) performance on average size judgment tasks is relatively unaffected by display time or set size (Ariely, 2001; Chong \& Treisman, 2003). However, the claim that these findings support the hypothesized average size perception mechanism is based on the assumption that people do the task by calculating the average. That is, the inference of a new size perception mechanism is based on the idea that people cannot calculate the average of all of the items in the array using focused attention. We agree that such a serial averaging of all the items in the display is implausible. Yet we have shown that people could perform these tasks by focusing attention on only a small subset of the items in the set. A strategy of randomly sampling one or two items is relatively unaffected by the number of items in the set (see, e.g., Simulation 1), and given that searching for one or two items can occur quickly, performance with this sort of strategy should also be relatively unaffected by display time.

That performance on a mean judgment task exceeds recognition performance for individual items would be surprising only if subjects were focusing attention on all the items in the set. However, if they focus only on a subset of items, recognition of many items in the set should be poor. Note that our sampling explanation might predict better performance for smaller set sizes, because fewer items would be unattended. That is, if observers perform the mean judgment task by sampling a small number of items and averaging those, they should also be able to recognize a sample from a small number of items with some degree of precision. The original Ariely (2001) study showed no effect of set size on the probability of labeling a test probe as a studied item (his data did not present sensitivity as a function of set size). In his task, the number of unique sizes in each display was constant, and the number of instances of each size was varied. Under such conditions, sampling produces relatively similar performance across set sizes of 8,12 , and 16 . Performance should be better with only 4 unique objects in the display, however, if subjects are able to encode and retain a subset of the items with good precision.

One possible reason for the absence of better performance at the smallest set size of four items was that performance was poor for all set sizes. The demands of the member recognition task might be more stringent than the demands of the mean judgment task: In the mean judgment task, observers need to retain only the result of the operation; in the member recognition task, they need to try to remember all of the individual items. More important, even if these two tasks do show different patterns of performance that reflect the operation of different mechanisms, both mechanisms might be based on focused attention, rather than on a new averaging mechanism.

Although we have shown that performance in average size judgment tasks is consistent with the limits on focused attention, we do not claim that subjects actually use the strategies we have identified (although anecdotally, subjects occasionally report such shortcuts during debriefing). And they might well have an average size perception mechanism that accomplishes the task. However, in contrast to claims in the literature, the evidence to date does not necessitate such a mechanism. We argue that it is simpler to explain performance via known mechanisms than to posit a new average size perception mechanism.

Several other inconsistencies in the average size perception literature raise further doubts about the existence of a global, parallel average size perception mechanism. First, performance across tasks varies substantially, from $4 \%-12 \%$ precision in an array/probe task to much less precise $(>20 \%)$ performance with a forced choice between two probes. If observers can perceive the mean size of an array of items at a glance, it is unclear why performance should vary so widely. Of course, these two tasks may require different levels of precision in the representation of size, but claims for averaging are often based on absolute levels of performance (i.e., good performance must reflect averaging), and in the absence of comparisons with what could be accomplished with focused attention, such variability makes it less clear what counts as good enough performance to support a claim of average size perception.

Another question is whether the purported averaging mechanism involves averaging of all of the elements in a set or just a subset of items that exceeds the limits of focused attention. All of the published research on the topic implies that averaging is of all items in the display, or that the information used to do the averaging is processed globally and in parallel. This implication is apparent from three aspects of the published research on the topic: (1) Much of the research explicitly states that the methods 
used would require averaging of all items; (2) most of the research is framed by the claim that average size perception might provide a summary representation of an entire display, much like the gist for a scene image; and (3) none of the research has explicitly examined how well subjects could perform without needing to focus attention on all the items in a display.

In principle, though, evidence for a new mechanism would not require averaging of all the items in the display. Rather, it would require just that performance exceed the limits of focused attention. For example, if performance could be explained only by averaging of more than about five items, presumably some new mechanism would be needed to explain performance. This logic is less demanding than requiring averaging of all the items in the display, but it is less clear what form such a mechanism would take and what purpose it would serve. The reason that claims of an average size perception mechanism are interesting is that such a mechanism might help to explain the rapid perception of scene gist or other global properties of a display. Yet, if performance is based on only a subset of the display, the mechanism would not be one that extracts global properties of the scene, so it would be less clear that it could generalize to phenomena such as scene gist perception.

Taken together, the results of our experiments and simulations suggest a more mundane explanation for the striking finding that observers can judge the average size of an array of objects. Rather than computing the mean of all elements in the array, subjects potentially could use strategies that rely on focused attention. We have identified a few such strategies and have shown that an ideal observer using those strategies can produce performance as good as that shown in the original studies. Other, more sophisticated strategies might lead to even better performance. Our evidence does not demonstrate that people do use such strategies, and it does not exclude the possibility that they can perceive the average size of the set. However, it does show that existing evidence does not necessitate a new, impressive cognitive ability. Performance is entirely consistent with the claim that judgments of average size in these tasks are subject to the established limits of focused attention.

\section{AUTHOR NOTE}

Experiments 1 and 2 were conducted as part of an undergraduate honors thesis by K.M., who is currently a neuroscience graduate student at the University of California, Irvine. K.M. and D.J.S. jointly designed those experiments, and K.M. was responsible for implementing, conducting, analyzing, and modeling them. He also wrote the initial draft of the manuscript, which did not include simulations of other averaging studies in the literature. D.J.S. was responsible for all of the other simulations and revisions of the manuscript. Thanks to Eamon Caddigan for help in programming the experiments. Thanks to Sang Chul Chong and Anne Treisman for providing additional details about their methods and for helpful feedback and discussion. Thanks also to Jan de Fockert, Dan Ariely, Brian Scholl, Mike Roy, and Alejandro Lleras for helpful comments. Correspondence concerning this article should be addressed to D. J. Simons, Department of Psychology, University of Illinois, 603 E. Daniel Street, Champaign, IL 61820 (e-mail: dsimons@uiuc.edu) or to K. Myczek, Department of Neurobiology and Behavior, University of California, Irvine, 1216 Gillespie, Irvine, CA $92697-4545$ (e-mail: kmyczek@uci.edu).

\section{REFERENCES}

ARIELY, D. (2001). Seeing sets: Representation by statistical properties. Psychological Science, 12, 157-162.

Cavanagh, P. (2001). Seeing the forest but not the trees. Nature Neuroscience, 4, 673-674.

Chong, S. C., \& Treisman, A. [M.] (2003). Representation of statistical properties. Vision Research, 43, 393-404.

Chong, S. C., \& Treisman, A. [M.] (2005a). Attentional spread in the statistical processing of visual displays. Perception \& Psychophysics, 67, 1-13.

Chong, S. C., \& Treisman, A. [M.] (2005b). Statistical processing: Computing the average size in perceptual groups. Vision Research, $\mathbf{4 5}, 891-900$.

DE Fockert, J. W., \& Marchant, A. P. (2008). Attention modulates set representation by statistical properties. Perception \& Psychophysics, 70, 789-794.

Oliva, A., \& Schyns, P. G. (2000). Diagnostic colors mediate scene recognition. Cognitive Psychology, 41, 176-210.

Oliva, A., \& Torralba, A. (2001). Modeling the shape of a scene: A holistic representation of the spatial envelope. International Journal of Computer Vision, 42, 145-175.

Parkes, L., Lund, J., Angelucci, A., Solomon, J. A., \& Morgan, M. (2001). Compulsory averaging of crowded orientation signals in human vision. Nature Neuroscience, 4, 739-744.

PotTer, M. C. (1976). Short-term conceptual memory for pictures. Journal of Experimental Psychology: Human Learning \& Memory, 2, 509-522.

Rensink, R. A. (2000). Seeing, sensing, and scrutinizing. Vision Research, 40, 1469-1487.

Rensink, R. A. (2004). Visual sensing without seeing. Psychological Science, 15, 27-32.

Sussman, R. S., \& Scholl, B. J. (2008). The flexibility of statistical summary representations in vision. Manuscript submitted for publication.

Treisman, A. M., \& Gelade, G. (1980). A feature-integration theory of attention. Cognitive Psychology, 12, 97-136.

Watamaniuk, S. N. J., \& Duchon, A. (1992). The human visual system averages speed information. Vision Research, 32, 931-941.

Wolfe, J. M., Alvarez, G. A., \& Horowitz, T. S. (2000). Attention is fast but volition is slow. Nature, 406, 691 .

\section{NOTES}

1. The hypothesis of a global, parallel averaging mechanism for size leaves open the question of what visual information underlies the average and how that information is processed in parallel across the display. One candidate for this information might be spatial frequency, which could potentially be used to derive something akin to object sizes in the display. However, spatial frequency filters would not explain averaging performance in studies using outline circles as stimuli. Another possibility is overall luminance; the display with a larger average might have an overall higher luminance. Note, though, that perceiving the side of the display with greater overall luminance has little to do with perceiving average size at all. And one reason for using outline circles is to minimize the use of luminance as a cue. In the absence of receptors specific to different absolute object sizes, it is unclear what global information could be processed in parallel across the display to generate a percept of average size.

2. Our simulations are not intended to show that people actually do use the strategies we propose or that people definitively do not perceive average size using a global, parallel mechanism. Rather, we try to show that focused-attention strategies potentially could explain all of the existing evidence. If such focused-attention strategies are sufficient to explain the existing evidence, the impetus for a new averaging mechanism is weakened. Further evidence might well demonstrate the insufficiency of such focused-attention explanations.

3. Since the initial preparation of this article, at least one article in the publication process has used the method of adjustment (Sussman 
$\&$ Scholl, 2008). The goal of that article was to compare average size perception for different types of objects, and it did not focus on distinguishing the levels of performance expected for focused attention, as opposed to a global, parallel mechanism. Interestingly, absolute performance levels for their task were not particularly accurate. For this article, we chose to examine only studies in print in the literature at the time we wrote our article, both because they are readily accessible and because they have made the strongest claims about the need for a new average size perception mechanism.

4. The original article (Ariely, 2001) did not make clear whether averaging was based on the diameters or the areas. Given that all values were reported in terms of diameters, we based our calculations on diameters as well (except where we explicitly note otherwise).

5. The de Fockert and Marchant (2008) experiments were conducted independently of ours, and each lab was made aware of the other's completed work through a mutual colleague. Both manuscripts were submitted simultaneously, with the goal that they be published as companion pieces in the same journal issue.
6. Some of the method details in the brief original article were insufficient to determine the exact object sizes, due to subtle inconsistencies in the descriptions of how they were generated. And unfortunately, the original algorithms used to generate the stimuli are no longer available. For example, with the small range, each item was $5 \%$ bigger than the next smallest item on a log scale, meaning that the items were evenly distributed on a log scale. However, the mean size of the array was said to be halfway between the largest and the smallest items, implying that the items were linearly distributed, rather than distributed on a log scale. We selected object sizes on the basis of the assumption that the endpoint sizes mentioned in the article were exact, and we interpolated between them on a log scale so that the items were evenly distributed. This approach yielded a multiplicative factor close to that described in the article, but the array mean on a nonlog scale differed slightly from the stated mean. We report the exact item diameters used in the simulation.

(Manuscript received November 14, 2006; revision accepted for publication August 10, 2007.) 